\title{
Antecedent reinforcement schedule training and operant response reinstatement in rats
}

\author{
GEORGE J. FRANKS and KENNON A. LATTAL \\ West Virginia University, Morgantown, West Virginia 26506
}

\begin{abstract}
Rats were trained on reinforcement schedules which generated high or low response rates. After extinguishing responding by eliminating food-reinforcement delivery, response-independent food presentations reinstated responding. Higher response rates occurred if the schedule preceding extinction controlled high response rates, suggesting that discriminative stimulus properties of the reinforcer were a function of antecedent training schedules.
\end{abstract}

A number of variables have been functionally related to responding maintained by schedules of response-independent reinforcement. Adventitious temporal contiguity of responding and reinforcement (Skinner, 1948) is probably the most common general account of the maintenance of such behavior. Other investigators have proposed alternative or complementary mechanisms which emphasize the discriminative properties of the reinforcement (Rescorla \& Skucy, 1969) or of the responsereinforcement relationship (Lattal, 1975).

One procedure for examining the role of a reinforcer in controlling responding through its discriminative properties was systematically described by Reid (1957). Animals were trained to respond to produce food. Upon removal of the food reinforcement (extinction), the response rate decreased to low levels. Reintroduction of response-independent presentations of food reinstated responding, but presentation of stimuli other than food (e.g., flashes of a light and/or presentation of a buzzer) did not reliably reinstate responding. Spradlin, Girardeau, and Hom (1966), Spradlin, Fixsen, and Girardeau (1969), and Campbell, Phillips, Fixsen, and Crumbaugh (1968) replicated Reid's (1957) findings using a somewhat different procedure in which delivery of the previously response-produced reinforcer was dependent upon the omission of responding for a specified interval. The initial reinforcer deliveries reinstated responding, but this effect diminished as the omission contingency gained control over the response. The presentation of other stimuli not paired with reinforcement had no systematic effect on responding.

Several parameters of the response-reinstatement effect have been investigated. Rescorla and Skucy

Appreciation is expressed to O. J. Sizemore, J. Bruner, and $V$. Nussear for their assistance in various stages of the experiment. Reprints may be obtained from K. A. Lattal, Department of Psychology, West Virginia University, Morgantown, West Virginia 26506.
(1969) extinguished rats' barpressing responses by omitting the delivery of food pellets after training with either response-dependent or responseindependent food presentations. Subsequent delivery of response-independent food pellets reinstated higher response rates in the group that received training with response-dependent food pellets. Rescorla and Skucy reported no systematic relation between the magnitude of the response-reinstatement effect and duration of intervening exposure to extinction. The response-reinstatement effect has been shown to be an increasing function of the number of training sessions with response-dependent reinforcement (Uhl, 1973). Topping, Pickering, and Jackson (1971) found no differences in responding during the delivery of response-independent reinforcers following extinction of responding previously reinforced according to either fixed or variable-interval schedules.

These studies show that reinforcing stimuli such as food and water may, like other stimuli, serve mulitple functions in the control of behavior. During schedules of response-independent reinforcement (cf. Zeiler, 1968), reinforcing stimuli are presented following a history of association with the behavior generated by a reinforcement schedule. If one source of control of responding during response-independent reinforcement schedules is the discriminative stimulus properties of the reinforcer, then reinforcing stimuli associated at different times with response-dependent reinforcement schedules which control different rates of responding should, following extinction of the response, control differential response reinstatement. The present experiment examined this possibility.

\section{METHOD}

\section{Subjects}

Three experimentally naive male hooded rats, 90 days old at the onset of the experiment, were maintained at $80 \%$ of freefeeding weight. 


\section{Apparatus}

A Scientific Prototype Model A-100 rodent test cage was equipped with a Davis Model 104 pellet dispenser that delivered standard 45-mg Noyes pellets as reinforcers. A $110-\mathrm{V}$ ac houselight provided continuous illumination during the session. The operandum, a Scientific Prototype Model PLS-100 lever, was operated by a minimal force of approximately $0.20 \mathrm{~N}$. The foodcup was located $7 \mathrm{~cm}$ (center to center) to the left of the lever and on the same chamber wall as the lever. The test cage was enclosed in a large chamber equipped with a ventilating fan and acoustical tile to mask extraneous noise. Control and recording equipment were located in an adjacent room.

\section{Procedure}

After each rat was trained to barpress, it was exposed to a sequence of three successive conditions: training on a schedule of reinforcement, extinction of barpressing (EXT, removal of food pellet delivery and accompanying stimuli) for several sessions, and then exposure to a fixed time schedule (FT 30-sec) in which reinforcers were delivered at 30 sec intervals independently of the animal's behavior. Two reinforcement schedules were used at different times during the training condition for each subject. A variable-ratio schedule (VR 20), in which, on the average, every 20th response was reinforced, was used to generate high response rates. A differential-reinforcement-of-low-rates schedule (DRL 30-sec), in which the first response after an absence of responding for $30 \mathrm{sec}$ was reinforced, was used to generate low response rates. The first FT 30-sec session was preceded by 30 min of extinction. This was done to preclude the adventitious reinforcement of any responses due to spontaneous recovery in the early portion of the first session. The first reinforcer during FT $30-\mathrm{sec}$ was never introduced within 10-15 min of a response (see Figures 2 and 3). This procedure was followed in only the first FT session after each extinction condition. In subsequent FT sessions, the first reinforcer was presented $30 \mathrm{sec}$ after the session started and subsequent reinforcers occurred at 30 -sec intervals during the session. The entire sequence of response-dependent reinforcer schedule training (either VR 20 or DRL $30 \mathrm{sec}$ ), EXT, and FT $30 \mathrm{sec}$ was repeated four times. The order and number of sessions in each experimental condition are provided in Table 1. After the first exposure to the DRL schedule, sessions generally lasted for $60-90 \mathrm{~min}$ and were conducted 7 days a week. Some variability in session duration occurred as the session duration was ad. justed to each animal's body weight to insure against excessive weight gain or loss.'

Training on the VR and DRL schedules of reinforcement continued until response rates appeared stable upon visual inspection. Extinction training continued until fewer than 25 responses per $60-\mathrm{min}$ session occurred in five successive sessions. The FT schedules were in effect for at least five sessions (except for rat A4 during the first FT following extinction of DRL responding). The FT schedule remained in effect beyond five sessions on several occasions to assess longer term effects.

\section{RESULTS}

Figure 1 summarizes the effects of the different schedules on responding for each subject. Characteristic schedule control during the training schedules occurred with all subjects, i.e., high response rates occurred during VR 20 and low response rates occurred during DRL $30 \mathrm{sec}$. Over several sessions, responding during extinction was reduced to near-zero rates after both VR 20 and DRL $30 \mathrm{sec}$. The introduction of response-independent reinforcement according to a FT 30 -sec schedule after extinction uniformly pro-

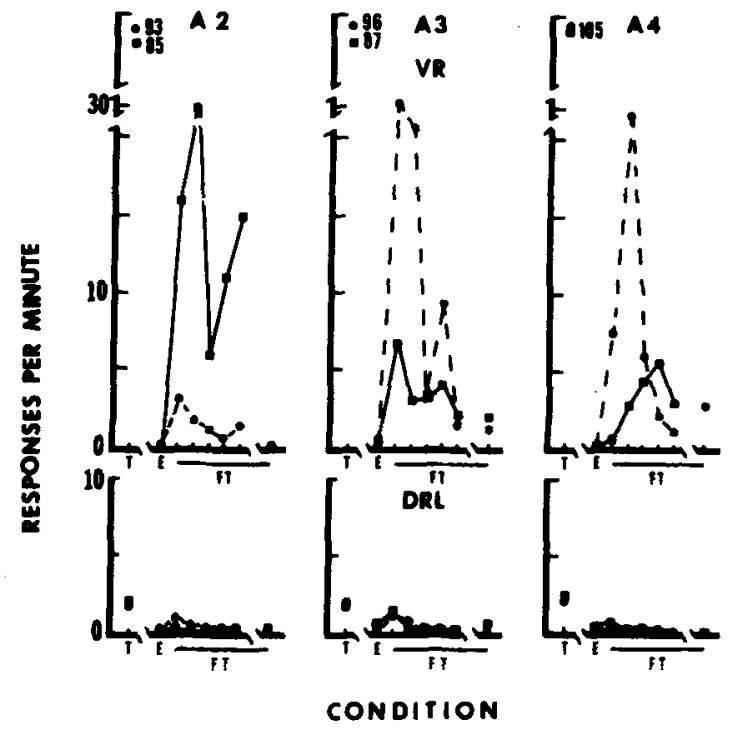

Figure 1. Responses per minute during the last training and extinction sessions and the first five and last sessions of FT $30 \mathrm{sec}$ during each sequence of experimental conditions. On the abscissa, $T$ indicates the last day of the training schedule; $E$ indicates the last day of extinction after the indicated training schedule. The sessions labeled FT are the first five and, where appropriate, the last FT sessions in the indicated sequence. The numbers beside the data points from $T$ (VR sequence) are the response rates during those sessions. The circles with dashed lines and squares with solid lines are, respectively, from the first and second exposure to each sequence of experimental conditions. VR and DRL sequences are, respectively, shown in the top and bottom sets of graphs.

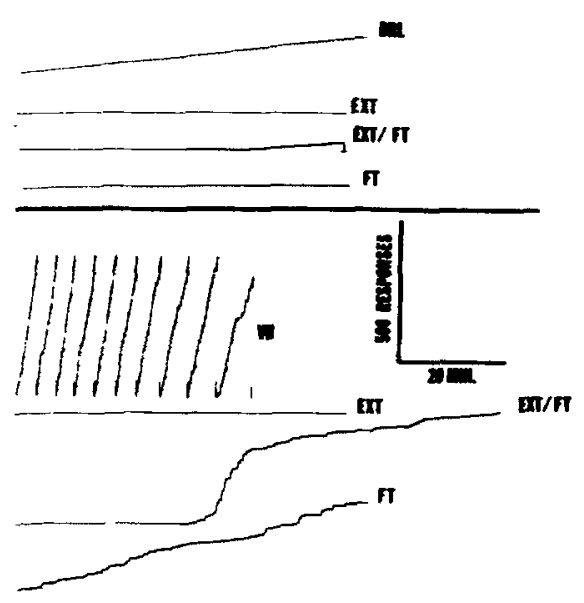

Figure 2. Cumulative records of subject $A 3$ during the last session of VR 20 and DRL 30 sec, and EXT after both training schedules. The first and last sessions of FT $30 \mathrm{sec}$ following VR 20 and DRL 30 sec training sessions are also shown. All records are from the second sequence of experimental conditions. Food pellet delivery is indicated by a downward deflection of the response pen. Because of an equipment failure, the first day of FT after extinction of VR responding lasted approximately 90 min instead of the usual $60 \mathrm{~min}$ (second record from the bottom of the figure). 


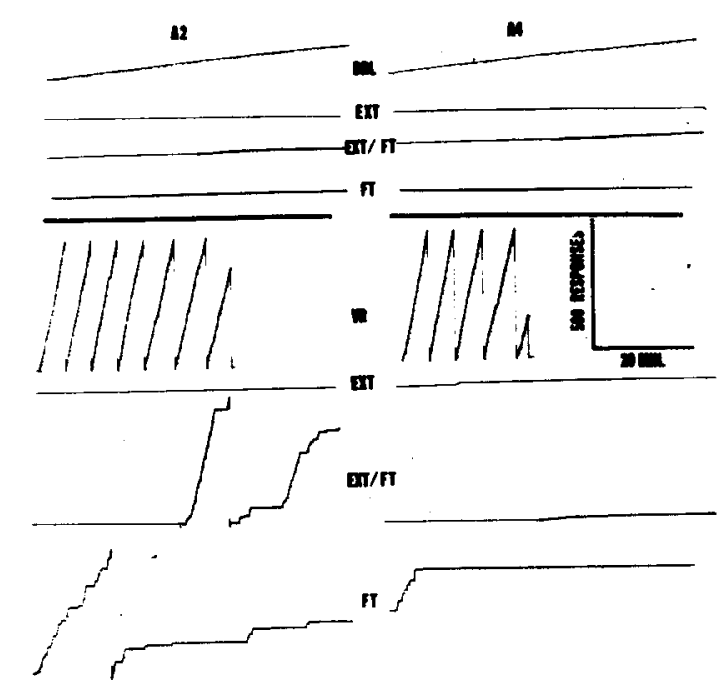

Figure 3. Cumulative records of subjects $A 2$ and $A$ during the last session of VR 20, DRL $30 \mathrm{sec}$, and EXT after both training schedules. The first and last sessions of FT $30 \mathrm{sec}$ following VR and DRL 30-sec training sessions are also shown. All records are from the second sequence of experimental conditions. Food pellet delivery is indicated by a downward deflection of the response pen.

duced higher response rates when the preceding training schedule was VR 20 than when it was DRL $30 \mathrm{sec}$. There was no systematic relation between duration of extinction and subsequent responding during the delivery of response-independent reinforcers. During FT 30 -sec conditions, the response rates generally declined both across and within sessions, although there was considerable variability in such behavior. The response patterns during the different schedules are illustrated for rat A 3 in Figure 2 and for rats A 2 and A 4 in Figure 3. Positively accelerated temporal response patterns of the type sometimes reported during exposure to FT schedules (Lattal, 1972; Zeiler, 1968) did not systematically occur during FT in this experiment.
Table 1 shows that the frequency of reinforcement was higher during the VR 20 schedule than during the DRL 30-sec schedule. Also, the frequency of reinforcement during FT $30 \mathrm{sec}$ was higher than that obtained during DRL $30 \mathrm{sec}$ and lower than that obtained during VR 20 in each sequence of schedules.

\section{DISCUSSION}

The response-independent delivery of a reinforcer (food) reinstated responding which was extinguished to near-zero rates following training on two different reinforcement schedules. Unlike the previously cited studies of response reinstatement effects, the present experiment showed that the response reinstatement magnitude was a function of the schedule under which the reinforcer was delivered prior to extinction. Cruse, Vitulli, and Dertke (1966) found that different types of reinforcers (standard and sucrose food pellets) served as discriminative stimuli controlling different operant responses. In this experiment, it is suggested that the same reinforcer reinstated different response rates because the reinforcer served as a discriminative stimulus which controlled different rates of responding as a result of association with the different training schedules.

While the present effects seem to be due primarily to these discriminative stimulus properties of the reinforcer, the operation of other variables should be considered. Order of presentation of the different training schedules does not seem to be crucial in accounting for these results, since the effects were replicated during the second exposure to each of the conditions. Neither duration of training on each reinforcement schedule nor duration of extinction training account for the obtained differences since neither was systematically related to response rates during the FT schedule. Differences in reinforcement

Table 1

Sequence of Experimental Conditions, Number of Sessions at Each Condition, Average Number of Reinforcers Delivered Per Minute, and Average Session Duration During the Last Five Sessions of Each Condition for Each Subject

\begin{tabular}{|c|c|c|c|c|c|c|c|c|c|}
\hline \multirow[b]{2}{*}{ Condition } & \multicolumn{3}{|c|}{ Rat A 2} & \multicolumn{3}{|c|}{ Rat A 3} & \multicolumn{3}{|c|}{ Rat A 4} \\
\hline & $\begin{array}{c}\text { Number } \\
\text { of } \\
\text { Sessions }\end{array}$ & $\begin{array}{c}\text { Reinforcers } \\
\text { per } \\
\text { Minute }\end{array}$ & $\begin{array}{l}\text { Session } \\
\text { Duration } \\
\text { (Minutes) }\end{array}$ & $\begin{array}{l}\text { Number } \\
\text { of } \\
\text { Sessions }\end{array}$ & $\begin{array}{c}\text { Reinforcers } \\
\text { per } \\
\text { Minute }\end{array}$ & $\begin{array}{c}\text { Session } \\
\text { Duration } \\
\text { (Minutes) }\end{array}$ & $\begin{array}{l}\text { Number } \\
\text { of } \\
\text { Sessions }\end{array}$ & $\begin{array}{c}\text { Reinforcers } \\
\text { per } \\
\text { Minute }\end{array}$ & $\begin{array}{c}\text { Session } \\
\text { Duration } \\
\text { (Minutes) }\end{array}$ \\
\hline DRL $30-\mathrm{sec}$ & 14 & 1.1 & 104.0 & 16 & 1.0 & 122.0 & 16 & 1.0 & 116.0 \\
\hline EXT & 14 & 0.0 & 60.0 & 15 & 0.0 & 60.0 & 9 & 0.0 & 59.0 \\
\hline FT $30-\sec$ & 5 & 2.0 & 60.0 & 7 & 2.0 & 59.0 & 4 & 2.0 & 55.4 \\
\hline VR 20 & 20 & 4.6 & 57.6 & 22 & 5.0 & 53.6 & 21 & 5.6 & 58.2 \\
\hline EXT & 20 & 0.0 & 60.0 & 27 & 0.0 & 87.8 & 21 & 0.0 & 66.2 \\
\hline FT 30-sec & 24 & 2.0 & 64.8 & 17 & 2.0 & 76.6 & 24 & 2.0 & 67.2 \\
\hline DRL $30-\mathrm{sec}$ & 10 & 1.1 & 64.6 & 10 & 1.3 & 81.4 & 10 & 1.0 & 87.2 \\
\hline EXT & 14 & 0.0 & 62.4 & 12 & 0.0 & 59.0 & 12 & 0.0 & 59.4 \\
\hline FT $30-\sec$ & 9 & 2.0 & 69.0 & 10 & 2.0 & 64.4 & 9 & 2.0 & 67.6 \\
\hline VR 20 & 10 & 4.2 & 22.4 & 10 & 5.3 & 51.8 & 10 & 5.8 & 14.6 \\
\hline EXT & 8 & 0.0 & 76.0 & 33 & 0.0 & 65.8 & 14 & 0.0 & 61.0 \\
\hline FT 30-sec & 5 & 2.0 & 70.0 & 7 & 2.0 & 63.2 & 5 & 2.0 & 55.8 \\
\hline
\end{tabular}


frequency between the training and FT schedules seem unlikely to account for the results, because the direction of the changes in response rates during FT 30-sec was opposite to that which would be predicted from a change in reinforcement frequency alone. That is, relative to the reinforcement frequency on the previous response-dependent schedule, the FT 30-sec schedule provided a lower reinforcement frequency after VR 20 and a higher reinforcement frequency after DRL $30 \mathrm{sec}$. Nevertheless, response rate was higher in the former case than in the latter.

The VR 20 and DRL 30-sec schedules also generated different frequencies of reinforcement as well as different response rates. That different reinforcement frequencies in the two training schedules alone account for the differences in magnitude of the response reinstatement after the two training schedules seems unlikely since similar reinstatement effects were obtained in both DRL sequences. These similar effects were obtained even though each animal had considerable exposure to more frequent reinforcement during the VR sequence which intervened between the first and second DRL 30-sec sequence.

Two alternatives to the account of the obtained effects in terms of the stimulus control of responding by the food pellet reinforcer are in terms of the disinhibition of extinguished responding and the adventitious reinforcement of barpressing. Previous demonstrations of the response reinstatement effect compared the effects of reinforcer presentation and the presentation of a "novel" or "neutral" stimulus. Each found that the reinstatement effect was controlled by the reinforcer and not by the disinhibition of responding in extinction by a stimulus change (Campbell et al., 1968; Reid, 1957; Spradlin et al., 1969). Although the present experiment did not include a control for such disinhibition effects, the operation of such a process here seems unlikely in light of the previous experimental evidence against a disinhibition interpretation of response reinstatement effects. Adventitious reinforcement cannot easily account for the initial response reinstatement effects. The probability of a response being reinforced by the initial response-independent pellet presentations was equal, since the response rates in the final extinction sessions were essentially equal. If the reinstated responding were simply due to adventitious reinforcement, the response rates following both types of training presumably would be the same.

Most studies of response-independent reinforcement begin by training the response according to a schedule of response-dependent reinforcement. During this training, the reinforcer is associated with schedule-controlled behaviors so that it may serve both a reinforcing and a discriminative function (cf. Cruse, Vitulli, \& Dertke, 1966). The rate at which responding is maintained when reinforcement occurs independently of responding has been related to several antecedent training schedule and reinforcement parameters. In addition, its effects are frequently attributed to the adventitious temporal contiguity of responding and reinforcement. However, in all such studies of response-independent reinforcement with which we are familiar, the same type of reinforcer has been used under both dependent and independent schedules, making it impossible to separate discriminative from other effects of reinforcement. Indeed, the total physical environment remains identical except for the removal of the response-reinforcement dependency. The present results suggest that rate of responding during schedules of response-independent reinforcement can be controlled through the discriminative stimulus properties of the reinforcer acquired during training with response-dependent reinforcement. The stimulus control exerted by the reinforcer during responseindependent reinforcement will, of course, change as a function of contemporary variables as well as by the passage of time since the reinforcement was associated with the training schedule. Although these discriminative stimulus properties of reinforcement probably do not fully account for the effects of response-independent reinforcement (cf. Rescorla \& Skucy, 1969, Experiment 4), the present results suggest that the role of this variable in such effects warrants further empirical and theoretical consideration.

\section{REFERENCES}

Campeell, P. A., Phillips, E., Fixsen, D., \& Crumbaugh, C. Free operant response reinstatement during extinction and time-contingent (DRO) reward. Psychological Reports, $1968,22,563-569$.

Cruse, D. B., Vitulli, W., \& Dertre, M. Discriminative and reinforcing properties of two types of food pellets. Journal of the Experimental Analysis of Behavior, 1966, 9, 293-303.

LATTAL, K. A. Response-reinforcer independence and conventional extinction after fixed-interval and variableinterval schedules. Journal of the Experimental Analysis of Behavior, 1972, 18, 133-140.

LAtTAL, K. A. Reinforcement contingencies as discriminative stimuli. Journal of the Experimental Analysis of Behavior, 1975, 23, 241-246.

REID, R. L. The role of the reinforcer as a stimulus. British Journal of Psychology, 1957, 49, 292-309.

REscorla, R. A., \& SxUCY, J. C. Effect of responseindependent reinforcers during extinction. Jourmal of Comparative and Physiological Psychology, 1969, 67, 381-389.

SKINNER, B. F. "Superstition" in the pigeon. Joumal of Experimental Psychology, 1948, 38, 168-172.

Spradlin, J. E., Fixsen, D. L., \& Girardeau, F. L. Reinstatement of an operant response by the delivery of reinforcement during extinction. Joumal of Experimental Child Psychology, 1969, 7, 96-100.

Spradlin, J. E., Girardeau, F. L., \& Hom, G. L. Stimulus properties of reinforcement during extinction of an operant 
response. Journal of Experimental Child Psychology, 1966, 4, 369-380.

Topping, J. S., Pickering, J. W., \& Jackson, J. A. Omission training effects following VI and FI pretraining. Psychonomic Science, 1971, 24, 113-114.

UHL, C. N. Eliminating behavior with omission and extinction after varying amounts of training. Animal Learning \& Behavior, 1973, 1, 237-240.

ZeILER, M. D. Fixed and variable schedules of responseindependent reinforcement. Joumal of the Experimental Analysis of Behavior, 1968, 11, 405-414.

\section{NOTE}

1. The first DRL condition was run longer in anticipation that the number of reinforcers delivered in DRL and VR would be equated. When the VR was run for $1 \mathrm{~h}$, the number of reinforcers was not equal to that in the previous DRL condition, so the session duration was maintained at between 60 and $90 \mathrm{~min}$ during succeeding conditions. The last VR condition for rats A 2 and A 4 was decreased in a further attempt to equate the number, but not the frequency, of reinforcement in the VR and DRL schedules.
(Received for publication April 18, 1975; revision accepted April 9, 1976.) 